\title{
Assessment of vitamin $E$ status in animals and man
}

By G. F. Comвs, Department of Poultry and Avian Sciences, and Division of Nutritional Sciences, Cornell University, Ithaca, New York, USA 14853

Almost 60 years ago, the nutritional essentiality of vitamin $E$ was first recognized (Evans \& Bishop, 1922). Since that discovery, numerous investigators using several species, have demonstrated nutritionally significant interrelationships between vitamin $\mathrm{E}$ and several other dietary components (e.g. selenium, polyunsaturated fatty acids, sulphur-containing amino acids, synthetic antioxidants). Despite the years of research, there has remained some disagreement concerning the biochemical mode(s) of action of the vitamin and hence, its role in human health. Within the last decade, however, discoveries concerning the metabolic nature of the well-known nutritional interrelationship of vitamin $\mathrm{E}$ and the essential element $\mathrm{Se}$ in farm and laboratory animals have provided the basis of a general hypothesis for the nutritional roles of vitamin $\mathrm{E}$ and related nutrients; that of maintenance of the functional integrity of subcelluar membranes. In such a role, vitamin $E$ is believed to have metabolic importance to all cells in the body, thus having fundamental significance in animal and human health.

\section{Role of vitamin $E$ in nutrition}

By the early I 970 s vitamin $\mathrm{E}$ had come to be regarded as a biologically specific antioxidant. Hypotheses concerning the basis for its established nutritional interrelationship with Se suggested either that both nutrients functioned as relatively nonspecific antioxidants, or that each had a different and specific function in normal metabolism. Indeed, the so-called Biological Antioxidant Hypothesis (Tappel, 1962) was supported by the fact that several vitamin E-deficiency diseases of animals are prevented by synthetic antioxidants, and that some evidence for increased lipid peroxidation in tissues from deficient animals could be produced. However, it was the discovery of the biochemical role of Se as a component of glutathione peroxidase (Rotruck et al. 1972) that stimulated this area of research, leading towards elucidation of the metabolic basis for the nutritional roles of vitamin $\mathrm{E}$ and Se.

Present understanding of this nutritionally important interrelationship is that vitamin $\mathrm{E}$ and $\mathrm{Se}$ are part of a multi-component antioxidant defence system within the cell which functions in protection from the adverse effects of reactive oxygen or free radical initiators of the oxidation of unsaturated phospholipids and critical proteins or both (Chow, I-979). Deleterious reactions against which this defence system must protect include the production of superoxide $\left(\mathrm{O}_{2}^{-}\right)$by the univalent reduction of $\mathrm{O}_{2}$ during metabolism. Disproportionation of $\mathrm{O}_{2}^{-}$is catalysed by 
superoxide dismutases which return half of the $\mathrm{O}_{2}$ and reduce the balance to $\mathrm{H}_{2} \mathrm{O}_{2}$ (Fridovich, 1975). Although $\mathrm{O}_{2}^{-}$and $\mathrm{H}_{2} \mathrm{O}_{2}$ can each directly promote lipid peroxidation (Demopoulous, 1963 ), the proximal agent in this process may be the hydroxyl radical $(\mathrm{OH})$ produced by reaction of these species according to the Fenton's reaction (McCord et al. I973). Vitamin E is known to be a good free radical scavenger (Urano \& Matsuo, I976). Therefore, the apparent role of vitamin $E$ is to remove these radicals as well as those produced by lipid peroxidation. Se, via glutathione peroxidase, is believed to have an analogous function by catalysis of the reduction of $\mathrm{H}_{2} \mathrm{O}_{2}$ (thereby reducing the production of $\mathrm{OH}$ ) or lipid hydroperoxides or both utilizing reducing equivalents from reduced glutathione (derived from the dietary sulphur-containing amino acids methionine and cystine). Thus, the metabolic function of vitamin $\mathrm{E}$ is intimately related to those of Se and other nutrients. Hence, it is appropriate to consider these functionally related nutrients in assessment of vitamin $E$ status.

\section{Diagnosis of vitamin $E$ deficiency}

Several vitamin E-related deficiency diseases have been clearly delineated in animals. The complex nutritional interrelationships of vitamin $\mathrm{E}$ and other dietary factors result in a multitude of acute and chronic deficiency disorders in several species (Table 1). These include disorders of the brain, cardiovascular system, liver, muscles and foetus. They may be categorized according to the nature of the nutritional basis for dysfunction; (I) disorders responsive to vitamin $E$ and synthetic antioxidants, (2) disorders responsive to vitamin E per se, (3) disorders responsive to vitamin $\mathrm{E}$, Se and sulphur-containing amino acids, (4) disorders responsive to Se per se.

The fact that several vitamin $\mathrm{E}$ deficiency diseases can be prevented by feeding synthetic antioxidants (i.e. ethoxyquin, DPPD, BHT) suggests that the biological antioxidant function of the vitamin, while metabolically important, may be rather nonspecific. Antioxidant-responsive disorders include foetal death and resorption in the rat, depigmentation of incisor enamel in the rat, and encephalomalacia in the chick. The fact that low concentrations of synthetic antioxidants are capable of preventing these diseases but fail to prevent other vitamin $\mathrm{E}$ deficiency syndromes indicates that in these tissues vitamin $\mathrm{E}$ acts as an antioxidant, a function which may also be performed by other appropriate antioxidants.

Vitamin $\mathrm{E}$ appears to have a more specific role in the prevention of a number of other syndromes. These include testicular degeneration and sterility in rats, rabbits, hamsters, guinea pigs, dogs, monkeys, and chickens. Vitamin E deficiency per se also results in several nutritional myopathies. These have been reviewed by Mason \& Horwitt (1972) and by Scott (1978). Myopathies responding primarily to vitamin $\mathrm{E}$ are seen as dystrophies of the striated muscles of vitamin E-deficient rats, guinea-pigs, rabbits, pigs and dogs. They are characterized by a hyaline, waxy, or Zenker's degeneration of muscle fibres, loss of striations, irregularities of sarcolemma nuclei, and vacuolation and swelling of sarcoplasm. The cardiac myopathy of the vitamin E-deficient pig, 'mulberry heart' (the pig also shows 


\section{Table $\mathrm{I}$. Known vitamin $E$ and selenium deficiency diseases of animals}

Disease

Reproductive failure

Foetal death, resorption Rat,

Testicular degeneration

Nutritional myopathies

Nutritional muscular dystrophy (NMD)

\section{NMD}

NMD

'Mulberry heart' disease

Gizzard myopathy

'Stiff lamb' disease

Creatinuria

Circulatory disorders

Erythrocyte hemolysis

Exudative diathesis

Anaemia

Other disorders

Liver necrosis

Membrane lipid peroxidation

Accumulation of ceroid

Kidney degeneration

Encephalomalacia

Incisor depigmentation

Pancreatic fibrosis
Animal

cow, ewe

Rooster, rat, rabbit, hamster, dog, pig, monkey

Chick ${ }^{*}$, rat, guinea-pig, rabbit, dog, monkey

Mink

Sheep, goat, calf

Pig

Turkey, duck

Newborn lamb

Rat, rabbit, guineapig, monkey

Chick, rat, rabbit

Chick

Monkey, pig

Rat, mouse, pig

Chick, rat

Rat, mink, calf, lamb, dog, chick, turkey

Mouse, rat, pig

Chick

Rat

Chick
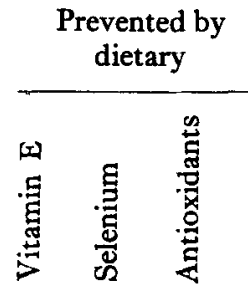

Tissue affected

Embryonic vascular system

Germinal epithelium

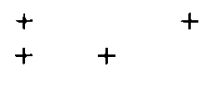

Striated muscle $\quad+\quad+t$

Striated cardiac muscle ++

Striated muscle +

Cardiac muscle

Gizzard muscle

Gizzard muscle

Striated muscle

Plasma

Plasma

Erythrocyte

Capillary walls

Bone marrow

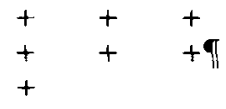

Liver

Hepatic mitochondria and microsomes

$\begin{array}{llll}\text { Adipose } & +\| & +\S & +\S \\ \text { Renal tubule contorti } & +\| 1 & & + \\ \text { Cerebellum } & +\| & & + \\ \text { Incisor enamel } & +\ddagger & & \\ \text { Pancreas } & & + & \end{array}$

"Responsive to sulphur-containing amino acids.

tMay partially reduce severity.

$¥$ Syndrome not easily produced in absence of dietary polyunsaturated fatty acids.

$\|$ Accelerated by polyunsaturated fatty acids.

$\S$ Involvement proposed but not confirmed.

Active only in the presence of selenium.

skeletal nutritional muscular dystrophy), is characterized by hydropericardium and myocardial hemorrhage. Monkeys and pigs also show anaemias as a result of vitamin $\mathrm{E}$ deficiency. 
Several diseases result from the combined deficiencies of vitamin E, Se, and in some cases the sulphur-containing amino acids. These include necrotic liver degeneration, nutritional muscular dystrophies, erythrocytic hemolysis, exudative diathesis in chicks, infertility in ewes and poor performance in several other farm animals.

The nutritional myopathies of lambs and calves are produced by feeding unsaturated fats in vitamin E- and Se-deficient diets. Vitamin E prevents these syndromes when they are associated with diets containing high levels of polyunsaturated fats, but the common nutritional muscular dystrophies of ruminants appear to be due primarily to Se deficiency. The combined deficiency of vitamin $\mathrm{E}$ and the sulphur-containing amino acids cystine and methionine produces in the skeletal muscles of the chick severe dystrophy which is only partially alleviated by Se. Se is integrally involved in protecting vitamin E-deficient fish from myopathy of skeletal muscles and in protecting the vitamin E-deficient turkey from gizzard and cardiac myopathies which do not respond to sulphur amino acids. These nutritional muscular dystrophies resemble histologically that observed in the duck, which in contrast, responds only to vitamin $\mathrm{E}$.

Combined vitamin $\mathrm{E}$ and Se deficiency in the chick results in exudative diathesis, an edematous condition of subcutaneous tissue resulting from abnormal permeability of capillary walls to plasma components. Severe exudative diathesis is fatal. The condition is not affected by the sulphur amino acid content of the diet, but it is alleviated in diets containing marginal levels of Se by reducing agents including ethoxyquin, ascorbic acid, and by high levels of vitamin $A$. The antioxidant effect has been shown to involve promotion of Se absorption. Some chlorinated hydrocarbons appear to interfere specifically with Se function, thus raising dietary requirements for the mineral.

Other vitamin $\mathrm{E}$ and Se deficiency syndromes have been reviewed by Scott (1978). These include infertility in ewes, foetal resorption in cows, impaired hair development in horses, impaired feather development in chickens, and poor general performance of cattle and sheep. The single disease that has been established to be a result of Se deficiency per se is the fibrotic degeneration of the exocrine pancreas in the chick. The dysfunctional pancreas has decreased production of pancreatic lipase, which results in reduced triglyceride digestion and impaired lipid-bile micelle formation necessary for the lumenal solubilization and absorption of lipids including vitamin $\mathrm{E}$. Therefore, secondary vitamin $\mathrm{E}$ deficiency due to impaired absorption is a consequence of the nutritional pancreatic atrophy produced by severe uncomplicated Se deficiency in the chick.

Controversy concerning the role(s) of vitamin $\mathrm{E}$ and related nutrients in human health results from (I) the lack (with a few exceptions) of clearly identified pathological changes strictly associated with low vitamin $\mathrm{E}$ nutriture and (2) numerous reports, many anecdotal in nature, of the efficacy of vitamin $\mathrm{E}$ therapy for several known diseases. Frank deficiencies of vitamin $E$ have generally been limited to premature infants and to patients with malabsorption syndromes. Despite the rise in maternal plasma tocopherol levels which occurs during 


\section{Vol. 40 Assessment of vitamin status in man and animals}

gestation the placental transfer of vitamin $\mathrm{E}$ to the foetus is apparently poor, as neonates normally have plasma tocopherol levels of only about one-third those of normal adults (Filer, 1968). When delivered prematurely, risk to vitamin $\mathrm{E}$ deficiency is further increased by impaired fat absorption resulting from the relative immaturity of the gastrointestinal tract. Several studies (i.e. Gordon $e t$ al. 1958; Gross \& Melhorn, 1972) have shown that plasma tocopherol levels of premature infants may not increase (and may even decrease) during the first $2-3$ months postpartum. Thus, the impaired utilization of dietary vitamin $\mathrm{E}$ by premature infants may place them at some risk when consuming prepared infant formula diets which, until recently, were relatively deficient in tocopherols as compared to human breast milk.

Associated with low circulating tocopherol levels in premature infants are several disorders that resemble those of vitamin E-deficient animals: hemolytic anaemia, hemorrhagic tendency, increased erythrocyte fragility (measured by induced hemolysis) and decreased erythrocyte survival (measured by ${ }^{51} \mathrm{Cr}$ labelling). These disorders have been reviewed by Farrell (1980). Although present evidence does not clearly establish direct causal relationships between these syndromes and 'chemical' vitamin $\mathrm{E}$ deficiency in the premature infants, knowledge obtained from animal experimentation strongly suggests that these syndromes may be signs of unprotected oxidative stress in the premature infant due to physiological vitamin $E$ deficiency. Support for this hypothesis comes from the observation that the placental transfer of Se to the foetus is also poor. (Rudolph \& Wong, 1978; Emerson et al. 1972), resulting in relative deficiency of the Se-dependent glutathione peroxidase in the neonate (Gordon et al. 1955).

Various types of gastrointestinal digestive or absorptive disturbances can also result in vitamin $\mathrm{E}$ deficiency in humans. Thus, vitamin $\mathrm{E}$ deficiency has been associated with gastrectomy, hepatic cirrhosis, cystic fibrosis (pancreatic involvement), chronic pancreatitis, pancreatic carcinoma, sprue, enteritis, ulcerative colitis, gluten enteropathy and biliary atresia. All of these disorders are associated to varying degrees with lipid malabsorption. In severe cases of vitamin E deficiency, patients show increased erythrocyte hemolysis, reduced erythrocyte survival, creatinuria and the deposition of ceroid in smooth muscles ('brown bowel'). However, such signs of vitamin $\mathrm{E}$ deficiency disease are rarely observed to be due to dietary inadequacies alone, and without associated steatorrhea. Although it is possible that chronic deficient vitamin E status may result in presently unrecognized pathological changes, especially in individuals with low Se intake, the symptomology of chronic vitamin $\mathrm{E}$ deficiency is not established. Reports critically reviewed by Scott ( 1978 ) provide evidence that nutritional or pharmacological levels of vitamin $\mathrm{E}$ may be important in helping to maintain body defences against thrombophlebitis, intermittent claudication, various eye diseases, dystrophic epidermolysis bullosa, gastric ulcers, liver disease, certain cancers and accumulation of ceroid. Plasma vitamin $E$ concentrations of at least $10 \mathrm{mg} / \mathrm{l}$ are required to optimally protect erythrocytes of man from oxidative hemolysis induced in vitro (Horwitt et al. 1956) and significant proportions of several 
populations have been found to have blood tocopherol levels less than one-half of that (plasma levels less than $5 \mathrm{mg} / \mathrm{l}$ are considered deficient). Surveys have indicated that as much as $\mathrm{r} \%$ of the Canadian general public (Desai, I968), $6 \%$ of Canadian university students (Desai, 1968) and $20-30 \%$ of East Pakistanis (Rhaman et al. 1964) had plasma vitamin E concentrations less than $5 \mu \mathrm{g} / \mathrm{l}$. Estimates of the average daily intake of vitamin $\mathrm{E}$ ( I I $-13.4 \mathrm{IU})$ and Se (98-224 $\mu \mathrm{g})$ in the United States indicate wide variations in the intake of these nutrients. It is likely, therefore, that many people may be of marginal status with respect to either or both of these nutrients as the result of such factors as abnormally high needs, poor absorption, and less than adequate daily intake.

\section{Methods of assessing vitamin $E$ status}

Vitamin E status is best assessed by tissue analysis of tocopherols and, in experimental circumstances, by evaluation by one or more tests of vitamin $\mathrm{E}$ function. Information so obtained is more useful if an assessment of Se status is also made either by tissue analysis of Se, or by measurement of its function, i.e. determination of Se-dependent glutathione peroxidase activity. Blood plasma or serum is the most useful tissue, especially for clinical use, in assessment of current vitamin E-Se status by biochemical analysis. Because plasma tocopherol levels are directly correlated with serum triglycerides (Farrell \& Bieri, 1975), it is useful to express results in terms of $\mathrm{mg}$ tocopherols/unit plasma lipids. In animal experimentation, analyses of liver or adipose tissue are valuable in estimation of total body reserves of vitamin $\mathrm{E}$. Tests of vitamin $\mathrm{E}$ function include the measurement of erythtocyte fragility by resistance to hemolysis in the presence of dialuric acid (Bruggemann et al. 1963) or $\mathrm{H}_{2} \mathrm{O}_{2}$ (Horwitt, 1960); measurement of liver storage of vitamin $\mathrm{E}$ (Bunnell, 1957); and measurement of protection against specific deficiency diseases such as nutritional muscular dystrophy in rabbits or chicks (Scott \& Desai, 1964; Fitch \& Diehl, 1965) encephalomalacia in chicks (Dam \& Sondergaard, I964), or exudative diathesis in Se-deficient chicks (Combs, 1978). The erythrocyte hemolysis and liver storage tests may be useful in the direct assessment of vitamin $\mathrm{E}$ status; all of these tests of vitamin $\mathrm{E}$ function may be employed as bioassays of vitamin $\mathbf{E}$ activity.

The analysis of vitamin $\mathrm{E}$ can be accomplished by several methods after extraction into an appropriate solvent and, for samples of high lipid content, saponification. Blood plasma or serum is extracted directly into hexane or xylene after alcoholic precipitation of the protein. Samples of tissues such as liver or adipose tissue are homogenized in chloroform-methanol and saponified using potassium hydroxide prior to extraction into hexane. The tocopherols and tocotrienols in lipid extracts can be separated by two-dimensional thin-layer chromatography on silica gel $\mathrm{G}$ using benzene-ethanol $(99: \mathrm{I} \mathrm{v}, \mathrm{v})$ as the first solvent, and hexane-ethanol ( $9: 1 \mathrm{v}, \mathrm{v})$ as the second solvent (Chow et al. 1969). Tocopherols can be visualized under ultraviolet light as spots of quenched fluorescence when fluorescein-treated plates are used, or by spraying with ferric chloride-bathophe nanthroline (Tsen, $196 \mathrm{I}$ ). 
The quantitative analysis of tocopherols in lipid extracts or in spots eluted from TLC plates is usually accomplished by a modification of the Emmerie \& Engel (1938) method which was based on the ability of tocopherols to reduce ferric chloride stoichiometrically to ferrous ions forming a coloured complex with $\alpha, \alpha^{\prime}$ dipyridine which was measured by its absorbance at $520 \mathrm{~nm}$. This method is substantially improved by the use of bathophenanthroline as the chromogenic reagent, and orthophosphoric acid to stabilize the colour by prevention of the photochemical reduction of the residual iron (Tsen, 196r). Spectrofluorometric analyses are more sensitive; such methods consist of measuring the fluorescence of tocopherol-containing extracts at their wavelengths of maximum excitation (295 $\mathrm{nm})$ and emission $(340 \mathrm{~nm})$ (Hansen \& Warwick, 1969). The sensitivity of this method has made fluorescence detection the method of choice in the measurement of tocopherols by high pressure liquid-liquid chromatography (Vatassery \& Hagen, 1977). Other procedures for tocopherol analysis (e.g. spectrophotometric, gas-liquid chromatographic and electrochemical methods) have value in food and pharmaceutical applications, however, they are not as convenient or sensitive as those outlined above, and are therefore less useful for routine analysis of biological materials in the assessment of vitamin $\mathrm{E}$ status.

Assessment of Se status is best accomplished by analysis of Se contents of whole blood, hair or liver, and by assay of the Se-dependent glutathione peroxidase in blood plasma or serum. Se can be determined by neutron activation analysis or by atomic absorption spectrometry; however, the method of choice for biological materials is a fluorometric method using diaminonapthalene (Olson et al. 1975). Se-dependent glutathione peroxidase can be assayed either by determination of residualnon-protein sulphydryls using Ellman's reagent after reaction of the sample with $\mathrm{H}_{2} \mathrm{O}_{2}$ and reduced glutathione (Rotruck et al. 1972); or by a kinetic procedure using the same substrates, coupling the reaction to glutathione reductase, and measuring the oxidation of NADPH as the change in absorbance at $340 \mathrm{~nm}$ (Paglia \& Valentine, I967).

The methods outlined above are useful in the assessment of vitamin E status, the full consideration of which must also involve assessment of Se status owing to the well characterized biochemical and nutritional interrelationships between the two nutrients. Such an approach will advance the elucidation of the biochemical bases for the nutritional functions of vitamin $\mathrm{E}$ and related nutrients, and will thus lead ultimately to a better understanding of the role of vitamin $E$ in the health of man and animals.

\section{REFERENCES}

Bruggeman, J., Nieser, K. H. \& Zentz, C. (1963). Int. Z. VitamForsch. 33, 180. Bunnell, R. H. (1957). Poultry Sci. 36, 4 I3.

Chow, C. K. (1979). Am. F. clin. Nutr. 32, 1066.

Chow, C. K., Draper, H. H. \& Csallany, A. S. (1969). Analyt. Biochem. 32, 81.

Combs, Jr., G. F. (1978). Poultry Sci. 57, 223.

Dam, H. \& Sondergaard, E. (1964). Z. Ernahrungswiss. 5, 73.

Demopoulous, H. B. (1963). Fedn Proc. Fedn Am. Socs exp. Biol. 32, 1859 . 
Desai, I. D. (1968). Can. F. Physiol. Pharmacol. 46, 819.

Emerson, P. M., Mason, D. Y. \& Cuthbert, J. E. (I972). Br. F. Haemat. 22, 667.

Emmerie, A. \& Engel, C. (I938). Rec. Trav. Chim. 57, I35 I.

Evans, H. M. \& Bishop, K. S. (I922). Science, N.Y. 56, 650.

Farrell, P. M. (1980). In Vitamin E: A Comprehensive Treatise, p. 520 [L. J. Machlin, editor]. New York: Marcell Dekker.

Farrell, P. M. \& Bieri, J. G. (1975). Am. f. clin. Nutr. 28, г381.

Filer, L. J. (1968). Am. F. clin. Nutr. 21, 3.

Fitch, C. D. \& Diehl, J. F. (1965). Proc. Soc. exp. Biol. Med. 119, 553.

Fridovich, I. (1975). Ann. Rev. Biochem. 44, 147.

Gordon, H. H., Nitowsky, H. M. \& Cornblath, M. (1955). Am. F. Dis. Child. 90, 669.

Gordon, H. H., Nitowsky, H. M., Tildon, J. T. \& Levin, S. (1958). Pediatrics, Springfield 21, 673.

Gross, S. \& Melhorn, D. K. (1972). Am. N.Y. Acad. Sci. 203, I4I.

Hansen, L. G. \& Warwick, W. (I969). Am. F. clin. Pathol. 51, 538.

Horwitt, M. K. (1960). Ann. F. clin. Nutr. 8, 451 .

Horwitt, M. K., Harvey, C. C., Duncan, G. D. \& Wilson, W. C. (1956). Am. F. clin. Nutr. 4, 404. McCord, J. M., Beauchamp, C. O., Guscin, S., Misra, H. P. \& Fridovich, I. (I973). In Oxidases and Related Redox Systems [King, T. E., Mason, H. S. \& Morrison, M., editors] pp. 58-66. Baltimore: University Park Press.

Mason, K. E. \& Horwitt, M. K. (1972). In The Vitamins: Chemistry, Physiology, Pathology, Methods, vol. 5, and ed. [Sebrell, W. H., Jr \& Harris, R. S., editors] pp. 272-292. New York: Academic Press.

Olson, O. E., Palmer, I. S. \& Cary, E. E. (1975). F. Ass. off. Analyt. Chem. 58, 117.

Paglia, D. E. \& Valentine, W. N. (1967). \%. Lab. clin. Med. 70, I 58.

Rhaman, M. M., Houssain, S., Talukdur, S. A., Ahmad, K. \& Bieri, J. E. (1964). Proc. Soc. exp. Biol. Med. I $7, \mathrm{I}_{33}$.

Rotruck, J. T., Pope, A. L., Ganther, H. E., Swanson, A. B., Hafeman, D. G. \& Hoekstra, W. G. (1972). Science, N.Y. 179, $5^{88 .}$.

Rudolph, N. \& Wong, S. L. (1978). Pediatric Res. 12, 789 .

Scott, M. L. (1978). In The Lipid Soluble Vitamins [DeLuca, H. F., editor] pp. 133-2ro. New York: Plenum Press.

Scott, M. L. \& Desai, I. D. (1964). F. Nutr. 83, 39.

Tappel, A. L. (1962). Vitams Horm. 20, 493.

Tsen, C. C. (196I). Analyt. Chem. 33, 849 .

Urano, S. \& Matsuo, M. (1976). Lipids 11, 380.

Vatassery, G. T. \& Hagen, D. F. (1977). Analyt. Biochem. 79, 29. 